\title{
Cirurgia de revascularização do miocárdio com e sem circulação extracorpórea. O que os novos estudos evidenciam?
}

\author{
Jhonatan Marchiori Ortolan ${ }^{\mathrm{a}^{*}}$, Lídia Trommer Marcos ${ }^{\mathrm{b}}$, Daniel Sturza ${ }^{\mathrm{c}}$, \\ Alexsandro Rodrigues de Oliveira ${ }^{\mathrm{a}}$
}

${ }^{a}$ Complexo Hospitalar Santa Casa de Misericórdia de Porto Alegre, Porto Alegre, RS, Brasil

${ }^{\text {bC }}$ urso de Biomedicina do Centro Universitário Ritter dos Reis - UniRitter, Porto Alegre, RS, Brasil

${ }^{c}$ Curso de Biomedicina da Universidade Federal do Rio Grande do Sul - UFRGS, Porto Alegre, RS, Brasil

Histórico do Artigo:

Recebido em:

02/12/2019

Aceito em:

$29 / 02 / 2020$

Palavras-chave:

Revascularização do miocárdio; circulação extracorpórea; CRM com CEC versus sem CEC

Keywords:

Myocardial revascularization; Cardiopulmonary bypass; On-pump versus Off-pump

\begin{abstract}
RESUMO
A doença arterial coronariana (DAC) é uma das principais causas de óbitos no mundo. A cirurgia de revascularização do miocárdio (CRM) prolonga a sobrevida e reduz a mortalidade de pacientes com DAC. Inúmeros estudos comparam os resultados obtidos em CRM com e sem o suporte da circulação extracorpórea (CEC), contudo, não existe um consenso sobre a modalidade ideal para esta cirurgia. $\mathrm{O}$ presente estudo consiste em uma revisão da literatura em que foram incluídas publicações que abordassem os resultados da realização de revascularização do miocárdio com e sem a utilização de circulação extracorpórea. Um total de 2.175.226 pacientes foram estudados, destes, 1.594.036 realizaram o procedimento com CEC e 581.190 foram submetidos a cirurgia sem CEC. A média de internação na unidade de tratamento intensivo (UTI) nos procedimentos realizados com CEC foi de 82,44 horas e quando realizado sem CEC foi de 55,95 horas. A média da quantidade de horas que os pacientes permaneceram sob ventilação mecânica após procedimentos com CEC foi de 16,12 horas e sem CEC foi de 11,34 horas. A mortalidade total dos pacientes submetidos a CRM com CEC foi de 909 sobre 25.074 indivíduos envolvidos (3,62\%). Quando o procedimento foi realizado sem CEC a mortalidade total foi de 771 pacientes de um total de 27.655 indivíduos operados $(2,84 \%)$. O número médio de enxertos que os pacientes receberam durante os procedimentos cirúrgicos foi maior no grupo que realizou o procedimento com CEC. Ocorreu da mesma forma, com relação à necessidade de transfusão sanguínea. É necessário que os pacientes sejam avaliados caso a caso, pois ambos os procedimentos, com e sem CEC, possuem vantagens e desvantagens. Não se pode considerar que uma técnica seja uniformemente superior a outra neste tipo de cirurgia.
\end{abstract}

Off-pump versus on-pump coronary artery bypass surgery. What do the new studies show?

\section{ABSTRACT}

Coronary artery disease (CAD) is one of the leading causes of death worldwide. Myocardial revascularization surgery prolongs survival and reduces mortality in patients with CAD. Numerous studies compare the results obtained in myocardial revascularization surgery with and without cardiopulmonary bypass (CPB), but there is no consensus on the ideal modality for this surgery. The present study consists of a literature review that included publications that addressed the results of myocardial revascularization with and without the use of cardiopulmonary bypass. A total of 2.175.226 patients were studied, of which 1.594.036 underwent the procedure with CPB and 581.190 underwent surgery without cardiopulmonary bypass. The average length of intensive care unit (ICU) stay in procedures performed with cardiopulmonary bypass was 82.44 hours and when performed without cardiopulmonary bypass was 55.95 hours. The average number of hours the patients remained under mechanical ventilation after CPB procedures was 16.12 hours and without cardiopulmonary bypass was 11.34 hours. The total mortality of patients undergoing cardiopulmonary bypass with CABG was 909 over 25,074 individuals involved $(3,62 \%)$. When the procedure was performed without cardiopulmonary bypass, the total mortality was 771 patients out of a total of 27,655 operated individuals $(2,84 \%)$. The average number of grafts that the patients received during the surgical procedures was higher in the group that underwent the procedure with cardiopulmonary bypass. It occurred the same way, regarding the need for blood transfusion. Patients need to be evaluated on a case-by-case basis, as both procedures, with and without cardiopulmonary bypass, have advantages and disadvantages. One technique cannot be considered to be uniformly superior to another in this type of surgery.

\footnotetext{
*Autor correspondente: jhonyortolan@gmail.com (Ortolan J.M.)
} 


\section{Introdução}

Considerada uma intercorrência grave de saúde, a doença arterial coronariana (DAC) é uma das principais causas de óbitos no mundo. Acredita-se que a DAC seja responsável por mais de $42 \%$ dos óbitos em pessoas acima de 65 anos (1).

A cirurgia de revascularização do miocárdio (CRM) prolonga a sobrevida e reduz a mortalidade de pacientes com DAC (2). Desde o início, o uso da circulação extracorpórea (CEC) tem sido o "padrão ouro" para a realização desse procedimento cirúrgico, proporcionando um campo cirúrgico estável e exangue. Entretanto, a CEC pode desencadear efeitos deletérios ao organismo como a resposta inflamatória sistêmica, depressão miocárdica, coagulopatias, instabilidade hemodinâmica, disfunção pulmonar, insuficiência renal e complicações neurológicas (3-5).

$\mathrm{Na}$ tentativa de reduzir as complicações pós-operatórias associadas a circulação extracorpórea, desenvolveu-se a técnica da CRM sem CEC, originalmente desenvolvida na década de 1960, porém, ganhando maior visibilidade e aplicabilidade em meados de 1990 com o desenvolvimento de estabilizadores cardíacos tornando a cirurgia sem CEC mais viável $(4,6-8)$. No entanto, a CRM sem bomba, como também é conhecida, igualmente possui efeitos prejudiciais ao organismo, dentre eles o baixo débito cardíaco intraoperatório, em alguns casos revascularização incompleta, assim, elevando taxas de morbimortalidade perioperatória $(7,9,10)$.

Inúmeros estudos comparam os resultados obtidos em CRM com e sem o suporte da circulação extracorpórea, contudo, não existe um consenso sobre a modalidade ideal para a revascularização cirúrgica do miocárdio. Dessa forma, devido ao fato de a literatura existente acerca desta temática ser bastante difusa, este trabalho tem por objetivo realizar uma revisão da bibliografia atual sobre o tema a fim de identificar qual método possui melhores resultados através de estudos recentes.

\section{Materiais e métodos}

O presente estudo consiste de uma revisão da literatura com base na busca de artigos científicos disponíveis nas bases de dados PubMed e Scielo, utilizando os descritores "coronary artery bypass", "on-pump versus off-pump", "myocardial revascularization". Foram incluídas publicações cuja temática abordassem os resultados da realização de revascularização do miocárdio com e sem a utilização de circulação extracorpórea em estudos publicados no período de 2014 a 2019, sendo a última pesquisa realizada na data de 31 de outubro de 2019, além de, obrigatoriamente, serem escritos na língua inglesa. Em um primeiro levantamento, 109 artigos foram selecionados. Primeiramente foram analisados títulos e resumos que contemplassem a temática sugerida e avaliados minuciosamente para que fossem incluídos. Foram excluídos artigos que contemplaram outros procedimentos cirúrgicos concomitantes, artigos publicados a mais de cinco anos, artigos que fizeram uso de minicircuito de perfusão (Mini-CEC) como técnica de perfusão, artigos de revisão bibliográfica e estudos que não realizaram comparação entre as duas técnicas cirúrgicas. De acordo com os critérios descritos anteriormente, foram selecionados e incluídos 33 estudos nesta revisão.

\section{Resultados}

Trinta e três artigos sobre a realização de cirurgia de revascularização do miocárdio com e sem uso de circulação extracorpórea, publicados no período de 2014 até 2019, foram utilizados para a realização desta revisão. Esse valor equivale à uma média de 5,5 artigos 
publicados por ano, conforme nossos critérios de inclusão.

Um total de 2.175.226 pacientes foram estudados nos artigos selecionados, destes, 1.594.036 realizaram o procedimento com CEC e 581.190 foram submetidos a cirurgia sem CEC. A tabela a seguir sumariza os trinta e três artigos incluídos nesta revisão da literatura, bem como, o número de pacientes envolvidos em cada estudo.

Tabela 1 - Artigos e quantidade de pacientes envolvidos em cada estudo.

\begin{tabular}{|c|c|c|c|c|}
\hline Autores & Artigos & $\begin{array}{c}\text { Quantidade } \\
\text { de Pacientes } \\
\text { Total }\end{array}$ & Com CEC & Sem CEC \\
\hline Davoodi et al., 2014 (19) & $\begin{array}{l}\text { Early outcome of off-pump } \\
\text { versus on-pump coronary } \\
\text { revascularization }\end{array}$ & 13.866 & 13.560 & 306 \\
\hline Ji et al., 2014 (24) & $\begin{array}{l}\text { On-pump versus off-pump } \\
\text { coronary artery bypass surgery } \\
\text { in high-risk patients: a } \\
\text { retrospective propensity score } \\
\text { matching analysis }\end{array}$ & 180 & 90 & 90 \\
\hline Lamy et al., 2014 (25) & $\begin{array}{l}\text { The cost implications of off- } \\
\text { pump versus on-pump coronary } \\
\text { artery bypass graft surgery at } \\
\text { one year }\end{array}$ & 4.752 & 2.377 & 2.375 \\
\hline Taggart et al., 2014 (37) & $\begin{array}{l}\text { Effects of on-pump and off- } \\
\text { pump surgery in the arterial } \\
\text { revascularization trial }\end{array}$ & 3.078 & 1.819 & 1.259 \\
\hline $\begin{array}{l}\text { Vieira de Melo et al., } 2014 \\
\text { (40) }\end{array}$ & $\begin{array}{l}\text { On-pump versus off-pump } \\
\text { coronary artery bypass surgery } \\
\text { in patients older than } 60 \text { years: } \\
\text { five-year follow-up of mass iii } \\
\text { trial }\end{array}$ & 176 & 86 & 90 \\
\hline Yu et al., 2014 (41) & $\begin{array}{l}\text { Off-pump versus on-pump } \\
\text { coronary artery bypass surgery } \\
\text { in patients with triple-vessel } \\
\text { disease and enlarged ventricles }\end{array}$ & 102 & 51 & 51 \\
\hline Bienz et al., 2015 (17) & $\begin{array}{c}\text { Microcirculatory response } \\
\text { during on-pump versus off- } \\
\text { pump coronary artery bypass } \\
\text { graft surgery }\end{array}$ & 32 & 16 & 16 \\
\hline Paparella et al., 2015 (27) & $\begin{array}{c}\text { On-pump versus off-pump } \\
\text { coronary artery bypass surgery } \\
\text { in patients with preoperative } \\
\text { anemia }\end{array}$ & 939 & 578 & 361 \\
\hline Ueki et al., 2015 (38) & $\begin{array}{c}\text { Off-pump versus on-pump } \\
\text { coronary artery bypass grafting } \\
\text { in patients with left ventricular } \\
\text { dysfunction }\end{array}$ & 2.187 & 1.134 & 1.053 \\
\hline Carmona et al., 2016 (18) & $\begin{array}{l}\text { Is off-pump technique a safer } \\
\text { procedure for coronary } \\
\text { revascularization? A propensity } \\
\text { score analysis of } 20 \text { years of } \\
\text { experience }\end{array}$ & 3.097 & 1.770 & 1.327 \\
\hline Hlavicka et al., 2016 (22) & $\begin{array}{c}\text { Off-pump versus on-pump } \\
\text { coronary artery bypass grafting } \\
\text { surgery in high-risk patients: } \\
\text { prague- } 6 \text { trial at } 30 \text { days and } 1 \\
\text { year }\end{array}$ & 206 & 108 & 98 \\
\hline Lamy et al., 2016 (26) & $\begin{array}{l}\text { Five-year outcomes after off- } \\
\text { pump or on-pump coronary- } \\
\text { artery bypass grafting }\end{array}$ & 4.752 & 2.377 & 2.375 \\
\hline Li et al., 2016 (1) & $\begin{array}{l}\text { Outcomes of off-pump versus } \\
\text { on-pump coronary artery bypass } \\
\text { graft surgery in patients with } \\
\text { severely dilated left ventricle }\end{array}$ & 50 & 24 & 26 \\
\hline
\end{tabular}




\begin{tabular}{|c|c|c|c|c|}
\hline Autores & Artigos & $\begin{array}{l}\text { Quantidade } \\
\text { de Pacientes } \\
\text { Total } \\
\end{array}$ & Com CEC & Sem CEC \\
\hline Singh et al., 2016 (34) & $\begin{array}{l}\text { On-pump versus off-pump } \\
\text { coronary artery bypass graft } \\
\text { surgery among patients with } \\
\text { type } 2 \text { diabetes in the bypass } \\
\text { angioplasty revascularization } \\
\text { investigation } 2 \text { diabetes trial }\end{array}$ & 615 & 444 & 171 \\
\hline Singh et al., 2017 (35) & $\begin{array}{l}\text { Renal function after off-pump } \\
\text { versus on-pump coronary artery } \\
\text { bypass grafting }\end{array}$ & 30 & 15 & 15 \\
\hline Friedrich et al., 2017 (21) & $\begin{array}{l}\text { On-pump versus off-pump } \\
\text { complete arterial } \\
\text { revascularization using bilateral } \\
\text { internal mammary arteries and } \\
\text { the t-graft technique: clinical } \\
\text { and angiographic results for } \\
\text { 3,445 patients in } 13 \text { years of } \\
\text { follow-up }\end{array}$ & 3.445 & 2.216 & 1.229 \\
\hline Benedetto et al., 2017 (13) & $\begin{array}{l}\text { Long-term survival after off- } \\
\text { pump versus on-pump coronary } \\
\text { artery bypass graft surgery. } \\
\text { Does completeness of } \\
\text { revascularization play a role? }\end{array}$ & 14.555 & 7.128 & 7.427 \\
\hline Shroyer et al., 2017 (32) & $\begin{array}{l}\text { Five-year outcomes after on- } \\
\text { pump and off-pump coronary- } \\
\text { artery bypass }\end{array}$ & 2.203 & 1.099 & 1.104 \\
\hline Yuksel et al., 2017 (10) & $\begin{array}{l}\text { A comparison of early clinical } \\
\text { outcomes of off-pump and on- } \\
\text { pump coronary artery bypass } \\
\text { grafting surgery in elderly } \\
\text { patients }\end{array}$ & 344 & 207 & 137 \\
\hline Amouzeshi et al., 2017 (11) & $\begin{array}{c}\text { Off-pump versus on-pump } \\
\text { coronary artery bypass graft } \\
\text { surgery outcomes during } 6 \\
\text { years: a prospective cohort } \\
\text { study }\end{array}$ & 61 & 21 & 40 \\
\hline Arslan et al., 2018 (12) & $\begin{array}{l}\text { Off-pump versus on-pump } \\
\text { complete coronary artery bypass } \\
\text { grafting comparison of the } \\
\text { effects on the renal damage in } \\
\text { patients with renal dysfunction }\end{array}$ & 94 & 48 & 46 \\
\hline Benedetto et al., 2018 (14) & $\begin{array}{l}\text { Comparison of outcomes for } \\
\text { off-pump versus on-pump } \\
\text { coronary artery bypass grafting } \\
\text { in low-volume and high-volume } \\
\text { centers and by low-volume and } \\
\text { high-volume surgeons }\end{array}$ & 2.094 .094 & 1.547 .851 & 546.243 \\
\hline Benedetto et al., 2018 (15) & $\begin{array}{l}\text { Off-pump versus on-pump } \\
\text { coronary artery bypass grafting. } \\
\text { Insights from the arterial } \\
\text { revascularization trial }\end{array}$ & 2.960 & 1.700 & 1.260 \\
\hline Scudeler et al., 2018 (31) & $\begin{array}{l}\text { Cost-effectiveness of on-pump } \\
\text { and off-pump coronary artery } \\
\text { bypass grafting for patients with } \\
\text { coronary artery disease: results } \\
\text { from the mass iii trial }\end{array}$ & 308 & 153 & 155 \\
\hline Shroyer et al., 2018 (33) & $\begin{array}{l}\text { Off-pump versus on-pump } \\
\text { impact: diabetic patient 5-year } \\
\text { coronary artery bypass clinical } \\
\text { outcomes }\end{array}$ & 835 & 433 & 402 \\
\hline
\end{tabular}




\begin{tabular}{|c|c|c|c|c|}
\hline Autores & Artigos & $\begin{array}{l}\text { Quantidade } \\
\text { de Pacientes } \\
\text { Total }\end{array}$ & Com CEC & Sem CEC \\
\hline $\begin{array}{l}\text { Parmeshwar et al., } 2018 \\
\text { (28) }\end{array}$ & $\begin{array}{l}\text { Off-pump versus on-pump: } \\
\text { long-term outcomes after } \\
\text { coronary artery bypass in a } \\
\text { veteran population }\end{array}$ & 555 & 238 & 317 \\
\hline Ulukan et al., 2019 (39) & $\begin{array}{l}\text { Comparisons of heart-type fatty } \\
\text { acid-binding protein (h-fabp) } \\
\text { levels in off-pump versus on- } \\
\text { pump coronary artery bypass } \\
\text { grafting }\end{array}$ & 30 & 15 & 15 \\
\hline Diegeler et al., 2019 (20) & $\begin{array}{c}\text { Five-year outcome after off- } \\
\text { pump or on-pump coronary } \\
\text { artery bypass grafting in elderly } \\
\text { patients }\end{array}$ & 2.187 & 1.134 & 1.053 \\
\hline Huang et al., 2019 (23) & $\begin{array}{l}\text { Late outcomes of off-pump } \\
\text { versus on-pump coronary } \\
\text { bypass in patients with diabetes: } \\
\text { a nationwide study from taiwan }\end{array}$ & 7.592 & 3.796 & 3.796 \\
\hline Soylu et al., 2019 (36) & $\begin{array}{l}\text { Comparison of intestinal } \\
\text { ischemia after on-pump versus } \\
\text { off-pump coronary artery bypass } \\
\text { grafting surgery }\end{array}$ & 7.606 & 1.210 & 6.396 \\
\hline Rocha et al., 2019 (29) & $\begin{array}{l}\text { Off-pump versus on-pump } \\
\text { coronary artery bypass grafting } \\
\text { in moderate renal failure }\end{array}$ & 3.156 & 1.578 & 1.578 \\
\hline Rufa et al., 2019 (30) & $\begin{array}{l}\text { Off-pump versus on-pump redo } \\
\text { coronary artery bypass grafting: } \\
\text { a propensity score analysis of } \\
\text { long-term follow-up }\end{array}$ & 216 & 108 & 108 \\
\hline \multirow[t]{2}{*}{ Benedetto et al., 2019 (16) } & $\begin{array}{l}\text { Off-pump versus on-pump } \\
\text { bypass surgery for left main } \\
\text { coronary artery disease }\end{array}$ & 923 & 652 & 271 \\
\hline & Total de Pacientes & 2.175 .226 & 1.594 .036 & 581.190 \\
\hline
\end{tabular}

A média de idade dos pacientes envolvidos nos estudos listados na Tabela 1 foi de 66,08 anos para os que realizaram procedimentos com CEC e de 65,97 anos para os que realizaram os procedimentos sem CEC.

Para a sequência da revisão, os artigos foram agrupados conforme dados que cada um abordava de forma específica. Dessa forma, a análise seguiu as seguintes abordagens: tempo (horas - h) de internação na UTI após CRM; horas até a extubação após o procedimento cirúrgico; mortalidade; número de enxertos recebidos; necessidade de transfusão sanguínea.

Sete artigos discorreram de forma específica a quantidade de horas que os pacientes permaneceram internados na UTI após a realização das cirurgias de revascularização do miocárdio com e sem circulação extracorpórea. A média de internação na UTI nos procedimentos realizados com CEC foi de 82,44 horas e quando realizado sem CEC foi de 55,95 horas, uma diferença média de 26,49 horas, ou seja, aproximadamente um dia de diferença. A figura 1 evidencia os artigos que fizeram essa comparação, bem como a média de horas de permanência na unidade de terapia intensiva após os procedimentos. 


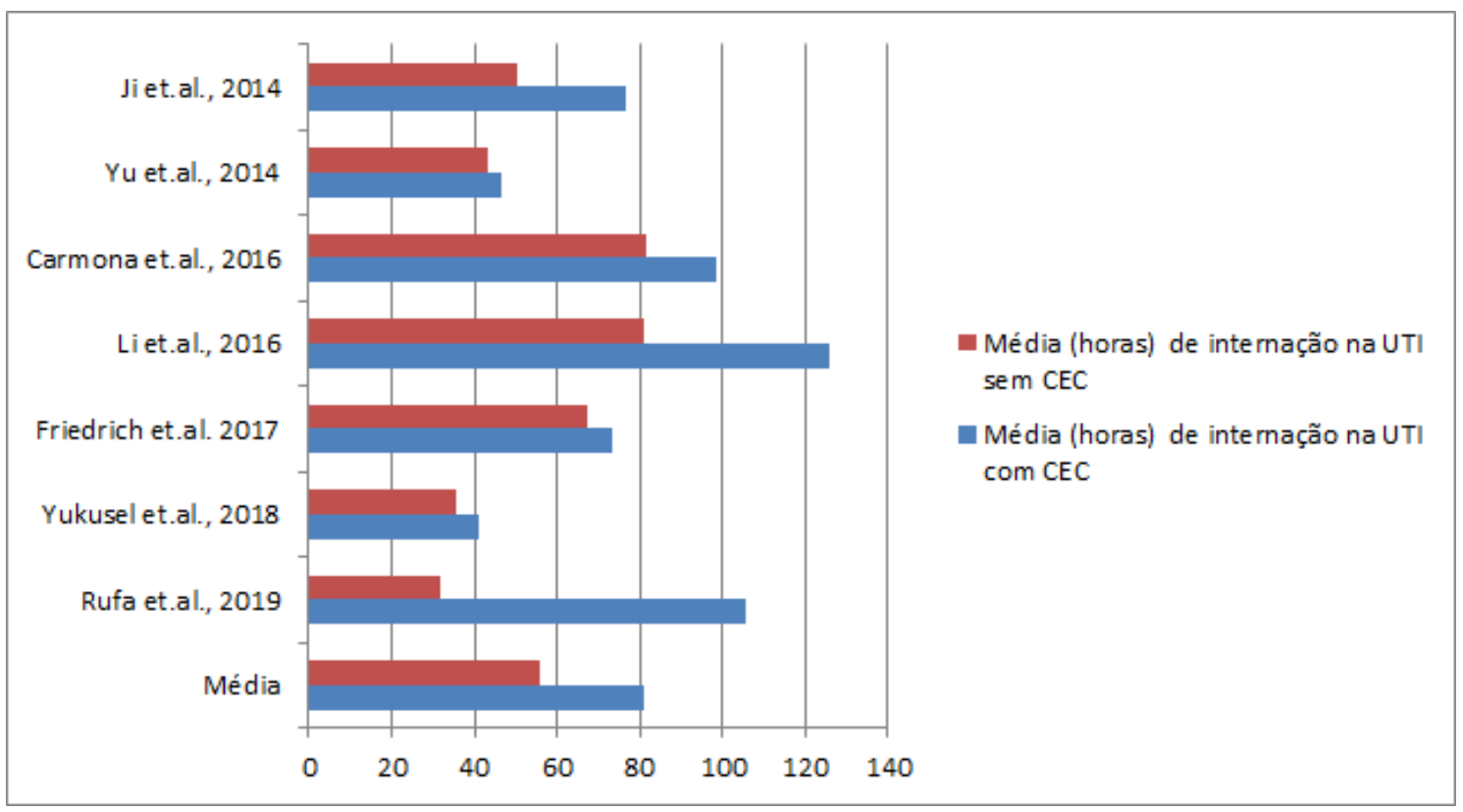

Figura 1 - Tempo (horas-h) de internação na UTI após CRM.

Seis artigos abordaram a quantidade de horas que os pacientes permaneceram sob ventilação mecânica após o procedimento cirúrgico. A média com CEC foi de 16,12 horas e sem CEC de 11,34 horas, uma diferença de 4,78 horas. A figura 2 evidencia os artigos que fizeram essa comparação e a média de horas até a extubação.

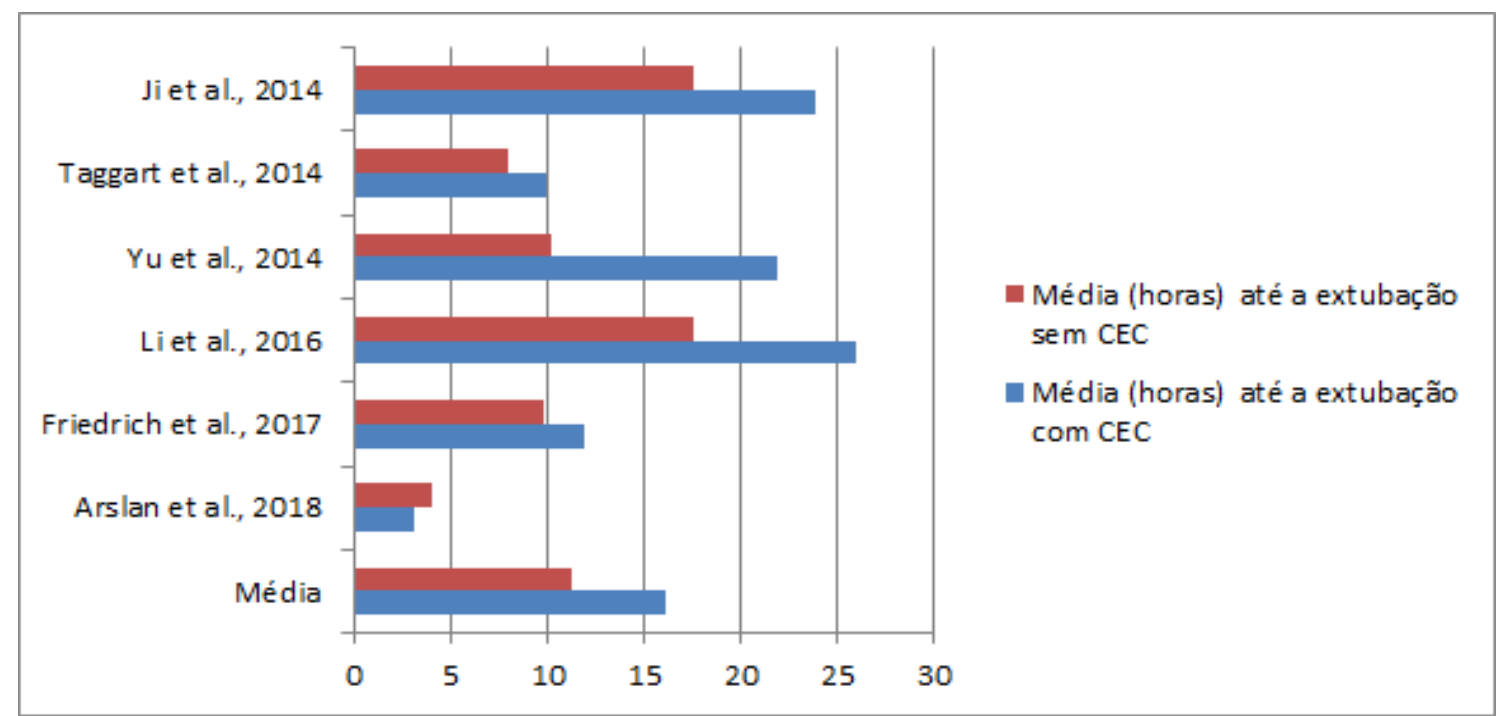

Figura 2 - Tempo (horas-h) da manutenção da respiração com a ventilação mecânica.

Com relação a mortalidade, 20 estudos relataram o número de óbitos, tanto no intraoperatório quanto no pós-operatório $(1,10,11,12,13,15,16,18,20,21,24,26,27$, $28,29,32,36,37,38,41)$. A mortalidade total dos pacientes submetidos a CRM com CEC foi de 909 sobre 25.074 indivíduos envolvidos (3,62\%). Quando o procedimento foi realizado sem CEC a mortalidade total nestes estudos foi de 771 pacientes de um total de 27655 indivíduos operados $(2,78 \%)$.

Treze dos trinta e três artigos abordaram o número médio de enxertos que os pacientes receberam durante os procedimentos cirúrgicos e, em todos treze estudos, o número de enxertos foi maior no grupo que realizou o procedimento com circulação extracorpórea, devido a complexidade dos casos, necessidades específicas de cada paciente e maior 
acessibilidade aos vasos coronarianos $(11,12,13,18,21,22,24,27,29,33,38,39,41)$. Da mesma forma, com relação à necessidade de transfusão sanguínea, o grupo com CEC demandou maior quantidade de transfusões sanguíneas em relação ao grupo sem CEC, um dos motivos seria a hemodiluição que ocorre com o uso da CEC. Estes dados referentes às transfusões sanguíneas estiveram presentes em onze dos trinta e três artigos selecionados $(10,11,12,16,19,20,21,29,37,38,41)$.

\section{Discussão}

Desde a década de 90, quando a cirurgia de revascularização do miocárdio sem uso de circulação extracorpórea passou a ter mais aplicabilidade, há uma intensa tentativa de comparar resultados clínicos da CRM com e sem CEC em curto e longo prazo. A CRM convencional (com CEC) é o processo geralmente realizado, porém, a perfusão extracorpórea pode representar uma total alteração da fisiologia normal do organismo. Alguns fatores como: hemodiluição, antigoagulação sistêmica, constantes variações de temperatura, trauma aos elementos figurados sanguíneos, fluxo sanguíneo não pulsátil e principalmente, a resposta inflamatória iniciada pelo contato do sangue com superfícies estranhas, possuem efeitos deletérios ao organismo $(3-5,9,10)$.

De forma geral a mortalidade peri e pós-operatória foi maior no grupo de pacientes que realizaram CRM com CEC. Porém, dos artigos que fizeram esta análise de mortalidade, três estudos verificaram a mortalidade em longo prazo (ao menos 5 anos após a realização da cirurgia) e, nestes estudos, foi constatada uma mortalidade superior no grupo de pacientes que realizaram o procedimento $\operatorname{sem} \operatorname{CEC}(11,28,32)$. Esta tendência de menor mortalidade a longo prazo observada nos pacientes que realizaram cirurgias com CEC pode estar intimamente relacionada com a quantidade de enxertos realizados nestes pacientes, uma vez que, todos os estudos que analisaram este ponto demonstraram que a quantidade de enxertos foi maior neste grupo de pacientes, ou seja, tiveram uma tendência maior à uma revascularização completa $(11,12,13,18,21,22,24,27,29,33,38,39,41)$.

É possível inferir que os pacientes que foram submetidos a CRM sem circulação extracorpórea tiveram uma recuperação pós-operatória mais rápida se for analisado o tempo médio até a extubação, bem como o tempo de estadia na unidade de tratamento intensivo. Este dado pode estar diretamente relacionado com a menor resposta inflamatória gerada quando o procedimento é realizado sem o suporte da CEC, pois, dessa forma, o sangue não é colocado em contato com as superfícies não endoteliais do sistema de circulação extracorpórea. Da mesma forma, o menor tempo cirúrgico, menor necessidade de hemodiluição, culminando na em menor necessidade de transfusões sanguíneas, igualmente pode influenciar na recuperação destes pacientes $(1,10,11,12$, $18,21,22,24,27,29,30,38,41)$.

A insuficiência renal (IR) ocorre em $30 \%$ dos pacientes que são submetidos a CRM e continua sendo uma grave complicação pós-operatória associada com significativos aumentos da morbimortalidade, sendo que o uso da circulação extracorpórea é um fator de risco para o desenvolvimento de IR (42, 43). Arslan et al (12), e Rocha et al (29), demonstraram em seus estudos com 3156 e 94 indivíduos, respectivamente, que os desfechos do procedimento sem CEC possui melhores resultados, diminuindo a incidência de lesão renal $(12,29)$. Por outro lado, Singh et al. (35), não evidenciou diferenças na função renal pós-operatória entre ambas as técnicas (35). Este resultado pode estar relacionado com o limitado número de pacientes envolvidos no estudo, no caso, trinta indivíduos presentes. Uma amostragem maior de pacientes poderia dar mais sustentabilidade à conclusão do autor em questão.

O risco de doenças cardiovasculares é de duas a quatro vezes maior em indivíduos com 
diabetes. Três artigos discorreram sobre a comparação entre as técnicas em pacientes diabéticos. Huang et al. 2019, conclui que em pacientes com diabetes, a realização da cirurgia de revascularização do miocárdio sem CEC ofereceu uma maior sobrevida no prazo de 30 dias, mas nenhuma diferença significante a longo prazo (23). Em contrapartida Shroyer et al. (33) e Singh et al. (34) demonstraram que os pacientes diabéticos submetidos a revascularização do miocárdio com CEC possuíam uma sobrevivência maior que o outro grupo, embora os resultados não sejam estatisticamente significantes $(33,34)$.

É sabido que a população idosa está aumentando nos últimos anos e isso tende a elevar o número de casos de DAC culminando na necessidade de revascularização do miocárdio. Esses indivíduos possuem um perfil de risco aumentado, associado a uma maior prevalência de comorbidades pré-operatórias e aumento da morbimortalidade peri e pósoperatórias. Nessa revisão não foram encontrados estudos que evidenciaram alguma diferença significativa entre pacientes idosos submetidos a CRM com ou sem CEC. Nos quatro estudos presentes nessa revisão, realizados com pacientes acima de 60 anos, os resultados foram semelhantes para os dois grupos, não evidenciando vantagens entre as técnicas $(10,20,28,40)$. Esses resultados se tornam controversos se analisarmos o desfecho da recuperação pós-operatória dos pacientes que realizaram o procedimento sem CEC, onde os mesmos possuem uma melhor recuperação pós cirurgia tendo como base os tempos de permanência na UTI e de manutenção da ventilação mecânica após os procedimentos $(1,10,12,18,21,24,30,37,41)$. Por outro lado, Soylu et al (36), comparando os resultados quanto à incidência de isquemia mesentérica pós revascularização do miocárdio, evidenciou que pacientes submetidos a CRM sem CEC possuem melhores desfechos comparados com os indivíduos operados com circulação extracorpórea (36).

Um estudo realizado por Benedetto et al. (16), envolvendo 999 centros de cirurgia cardíaca e um total de 2.094.094 pacientes, comparou os resultados de CRM com e sem CEC procurando evidenciar diferenças nos desfechos dos procedimentos realizados por cirurgiões que possuíam uma maior experiência no procedimento sem bomba versus cirurgiões que eram menos experientes nessa técnica, bem como os resultados de centros que realizavam com maior e menor frequência o procedimento sem CEC. O grupo concluiu que, para procedimentos sem CEC, os resultados são claramente dependentes do volume cirúrgico, tanto do centro de cirurgia cardíaca como do cirurgião individual (14). Esse estudo é de grande relevância, pois infere que, os resultados pós-operatórios da CRM sem CEC estão intimamente relacionados com a acurácia do cirurgião, sendo que serão positivos, se realizados por uma equipe experimente neste procedimento.

Com relação aos custos da realização de um procedimento de alta complexidade, como é a cirurgia cardíaca, na grande maioria das vezes, os mesmos são extremamente elevados. Este assunto foi exposto por dois dos trinta e três artigos analisados, onde os mesmos fizeram a comparação entre os dispêndios de CRM com e sem CEC $(25,31)$.

Em ambos os estudos a diferença financeira do procedimento em si não pôde ser considerada estatisticamente relevante, sendo assim, o custo do procedimento em si não deveria ser uma variável a ser levada em consideração quando da escolha da técnica a ser utilizada.

\section{Considerações finais}

O desenvolvimento da perfusão extracorpórea foi um grande avanço na cirurgia cardíaca, sem sua a utilização não seria possível a realização de milhares de procedimentos cirúrgicos feitos desde a sua descoberta até hoje.

A contribuição desta técnica para a medicina é imensurável, seja na cirurgia cardíaca, 
transplantes, infusão de quimioterápicos, etc. No entanto, sua utilização deve ser executada com cautela.

O principal exemplo que traz dúvida quanto ao uso ou não de CEC é a cirurgia de revascularização do miocárdio, este foi e continua sendo um assunto controverso. É necessário avaliar os pacientes caso a caso, pois ambos os procedimentos, com e sem CEC, possuem vantagens e desvantagens. Para isso, deve-se considerar e avaliar diversas variáveis de forma que seja escolhida a melhor técnica para cada paciente, uma vez que, até hoje, nenhum estudo comprovou efetivamente que uma técnica seja superior a outra. É importante esclarecer que o nível de gravidade dos pacientes submetidos às cirurgias interfere diretamente nos resultados, dessa forma, torna-se uma tarefa complicada realizar a comparação entre as duas técnicas.

Profissionais menos experientes podem dar preferência pelo procedimento de CRM com CEC por exigir menos destreza ao manuseio do coração, todavia, a circulação extracorpórea deve ser exercida com ponderação e todos seus possíveis efeitos deletérios devem ser levados em conta. Sendo assim, não se pode considerar que uma técnica seja uniformemente superior a outra neste tipo de cirurgia.

\section{Referências}

1. Li S, Gong W, Qi Q, Yuan Z, Chen A, Liu J, et al. Outcomes of off-pump versus on-pump coronary artery bypass graft surgery in patients with severely dilated left ventricle. Ann Transl Med 2016; 4(18): 340.

2. Yusuf S, Zucker D, Passamani E, Peduzzi P, Takaro T, Fisher LD, et al. Effect of coronary artery bypass graft surgery on survival: overview of 10-year results from randomised trials by the Coronary Artery Bypass Graft Surgery Trialists Collaboration. Lancet 1994; 344: 563-70.

3. Dieberg G, Smart NA, King N. On- vs. off-pump coronary artery bypass grafting: A systematic review and meta-analysis. Int J Cardiol 2016; 223:201-11.

4. Lemma MG, Coscioni E, Tritto FP, Centofanti P, Fondacone C, Salica A, et al. On-pump versus offpump coronary artery bypass surgery in high-risk patients: Operative results of a prospective randomized trial (on-off study). J Thorac Cardiovasc Surg 2012; 143(3): 625-31.

5. Edmunds LH. Inflammatory response to cardiopulmonary bypass. Ann Thorac Surg 1998; 66 (5 Suppl): S12-6.

6. Borst C, Jansen EWL, Tulleken CAF, Gründeman PF, Mansvelt Beck HJ, Van Dongen JWF, et al. Coronary artery bypass grafting without cardiopulmonary bypass and without interruption of native coronary flow using a novel anastomosis site restraining device ("Octopus"). J Am Coll Cardiol 1996; 27(6): 1356-64.

7. Ascione R, Angelini GD. Off-pump coronary artery bypass surgery: The implications of the evidence. J Thorac Cardiovasc Surg. 2003;125(4):779-81.

8. Rodrigues AJ, Évora PRB, Tubino PVA. Revascularização cirúrgica do miocárdio com versus sem circulação extracorpórea. O que mostram as evidências? Brazilian J Cardiovasc Surg 2013; 28(4): 5317.

9. Saba Davit, Gören Suna, Tekin Hakan, Aytaç Irem et al. The effects of position, ischemia and reperfusion to hemodynamics on the beating heart coronary bypass. Turkish J Thorac Cardiovasc Surg 2003; 11:26-31.

10. Yuksel A, Yolgosteren A, Kan II, Cayir MC, Velioglu Y, Yalcin M, et al. A comparison of early clinical outcomes of off-pump and on-pump coronary artery bypass grafting surgery in elderly patients. Acta Chir Belg 2017; 118(2): 99-104.

11. Amouzeshi A, Amouzeshi Z, Teshnizi MA, Moeinipour AA, Maleki MH. Off-pump versus on-pump coronary artery bypass graft surgery outcomes during 6 years: A prospective cohort study. Acta Med Iran 2017; 55(9): 578-84.

12. Arslan U, Calik E, Tekin AI, Erkut B. Off-pump versus on-pump complete coronary artery bypass grafting. Medicine (Baltimore) 2018; 97(35): 11-4. 
13. Benedetto U, Caputo M, Patel NN, Fiorentino F, Bryan A, Angelini GD. Long-term survival after offpump versus on-pump coronary artery bypass graft surgery. Does completeness of revascularization play a role? Int J Cardiol 2017; 246: 32-6.

14. Benedetto U, Lau C, Caputo M, Kim L, Feldman DN, Ohmes LB, et al. Comparison of Outcomes for Off-Pump Versus On-Pump Coronary Artery Bypass Grafting in Low-Volume and High-Volume Centers and by Low-Volume and High-Volume Surgeons. Am J Cardiol 2018; 121(5): 552-7.

15. Benedetto U, Altman DG, Gerry S, Gray A, Lees B, Flather M, et al. Off-pump versus on-pump coronary artery bypass grafting: Insights from the Arterial Revascularization Trial. J Thorac Cardiovasc Surg 2018; 155(4): 1545-1553.

16. Benedetto U, Puskas J, Kappetein AP, Brown WM, Horkay F, Boonstra PW, et al. Off-Pump Versus On-Pump Bypass Surgery for Left Main Coronary Artery Disease. J Am Coll Cardiol 2019; 74(6): 72940.

17. Bienz M, Drullinsky D, Stevens LM, Bracco D, Noiseux N. Microcirculatory response during on-pump versus off-pump coronary artery bypass graft surgery. Perfusion 2015; 31(3): 207-15.

18. Carmona P, Paredes F, Mateo E, Mena-Durán A V., Hornero F, Martínez-León J. Is off-pump technique a safer procedure for coronary revascularization? A propensity score analysis of 20 years of experience. Interact Cardiovasc Thorac Surg 2016; 22(5): 612-9.

19. Davoodi S, Karimi A, Ahmadi SH, Marzban M, Movahhedi N, Abbasi K, et al. Early outcome of offpump versus on-pump coronary revascularization. Pan Afr Med J 2014; 17: 1-6.

20. Diegeler A, Börgermann J, Kappert U, Hilker M, Doenst T, Böning A, et al. Five-Year Outcome After Off-Pump or On-Pump Coronary Artery Bypass Grafting in Elderly Patients. Circulation 2019; 139(16): 1865-71.

21. Friedrich C, Heller S, Cramer E, Awwad N, Amin W, Hansen L, et al. On-Pump versus Off-Pump Complete Arterial Revascularization Using Bilateral Internal Mammary Arteries and the T-Graft Technique: Clinical and Angiographic Results for 3,445 Patients in 13 Years of Follow-Up. Cardiol 2017; 136(3): 170-9.

22. Hlavicka J, Straka Z, Jelinek S, Budera P, Vanek T, Maly M, et al. Off-pump versus on-pump coronary artery bypass grafting surgery in high-risk patients: PRAGUE-6 trial at 30 days and 1 year. Biomed Pap 2016; 160(2): 263-70.

23. Huang KC, Wu IH, Chou NK, Yang YY, Lin LC, Yu HY, et al. Late outcomes of off-pump versus onpump coronary bypass in patients with diabetes: A nationwide study from Taiwan. $\mathrm{J}$ Thorac Cardiovasc Surg 2019; 157(3): 960-969.

24. Ji Q, Mei Y, Wang X, Ding W. On-Pump Versus Off-Pump Coronary Artery Bypass Surgery in HighRisk Patients. A Retrospective Propensity Score Matching Analysis. Int Hear J Assoc 2014; 55(1): 484-8.

25. Lamy A, Tong W, Devereaux PJ, Gao P, Gafni A, Singh K, et al. The cost implications of off-pump versus on-pump coronary artery bypass graft surgery at one year. Ann Thorac Surg 2014; 98(5): 16205.

26. Lamy A, Devereaux PJ, Prabhakaran D, Taggart DP, Hu S, Straka Z, et al. Five-year outcomes after off-pump or on-pump coronary-artery bypass grafting. N Engl J Med 2016; 375(24): 2359-68.

27. Paparella D, Guida P, Scrascia G, Fanelli V, Contini M, Zaccaria S, et al. On-pump versus off-pump coronary artery bypass surgery in patients with preoperative anemia. J Thorac Cardiovasc Surg 2015; 149(4): 1018-1026.

28. Parmeshwar N, Fero KE, Manecke G, Coletta JM. Off-Pump Versus On-Pump: Long-Term Outcomes After Coronary Artery Bypass in a Veteran Population. J Cardiothorac Vasc Anesth 2018; 33(5): 118794.

29. Rocha R V., Yanagawa B, Hussain MA, Tu J V., Fang J, Ouzounian M, et al. Off-pump versus onpump coronary artery bypass grafting in moderate renal failure. J Thorac Cardiovasc Surg 2020; 159(4):1297-1304.

30. Rufa MI, Ursulescu A, Nagib R, Shanmuganathan S, Albert M, Reichert S, et al. Off-pump versus onpump redo coronary artery bypass grafting: A propensity score analysis of long-term follow-up. J Thorac Cardiovasc Surg 2019; S0022-5223(19): 30971-7.

31. Scudeler TL, Hueb WA, Farkouh ME, Maron DJ, de Soárez PC, Campolina AG, et al. Costeffectiveness of on-pump and off-pump coronary artery bypass grafting for patients with coronary 
artery disease: Results from the MASS III trial. Int J Cardiol 2018; 273: 63-8.

32. Shroyer AL, Hattler B, Wagner TH, Collins JF, Baltz JH, Quin JA, et al. Five-year outcomes after onpump and off-pump coronary-artery bypass. N Engl J Med 2017; 377(7): 623-32.

33. Shroyer ALW, Quin JA, Wagner TH, Carr BM, Collins JF, Almassi GH, et al. Off-Pump Versus OnPump Impact: Diabetic Patient 5-Year Coronary Artery Bypass Clinical Outcomes. Ann Thorac Surg 2018; 107(1): 92-8.

34. Singh A, Schaff H V., Mori Brooks M, Hlatky MA, Wisniewski SR, Frye RL, et al. On-pump versus off-pump coronary artery bypass graft surgery among patients with type 2 diabetes in the bypass angioplasty revascularization investigation 2 diabetes trial. Eur J Cardio-thoracic Surg 2016; 49(2): 406-16.

35. Singh RS, Thingnam SKS, Mishra AK, Verma I, Kumar V. Renal function after off-pump versus onpump coronary artery bypass grafting. Asian Cardiovasc Thorac Ann 2017; 25(7-8): 504-8.

36. Soylu L, Aydin OU, Yildiz M, Serdaroğlu H, Kurtoğlu M, Karademir S. Comparison of intestinal ischemia after on-pump versus off-pump coronary artbypass grafting surgery. Turkish J Med Sci 2019; 49(1): 11-5.

37. Taggart DP, Altman DG, Gray AM, Lees B, Nugara F, Yu LM, et al. Effects of on-pump and off-pump surgery in the arterial revascularization trial. Eur J Cardio-thoracic Surg 2014; 47(6): 1059-65.

38. Ueki C, Miyata H, Motomura N, Sakaguchi G, Akimoto T, Takamoto S. Off-pump versus on-pump coronary artery bypass grafting in patients with left ventricular dysfunction. J Thorac Cardiovasc Surg. 2015; 151(4): 1092-8.

39. Ulukan MO, Ugurlucan M, Unal O, Y1lmaz MF, Kasifoglu N, Sevin MB. Comparisons of heart-type fatty acid-binding protein (H-FABP) levels in off-pump versus on-pump coronary artery bypass grafting. Arch Med Sci - Atheroscler Dis 2019; 4(1):119-25.

40. Vieira de Melo RM, Hueb W, Rezende PC, Lima EG, Hueb AC, Ramires JAF, et al. On-pump versus off-pump coronary artery bypass surgery in patients older than 60 years: Five-year follow-up of MASS III trial. J Cardiothorac Surg 2014; 9(1): 1-5.

41. Yu L, Gu T, Shi E, Wang C, Fang Q, Yu Y, et al. Off-pump versus on-pump coronary artery bypass surgery in patients with triple-vessel disease and enlarged ventricles. Ann Saudi Med 2014; 34(3): 2228.

42. Chertow GM, Lazarus JM, Christiansen CL, Cook EF, Hammermeister KE, Grover F, et al. Preoperative renal risk stratification. Circulation 1997; 95: 878-84.

43. Chertow GM, Levy EM, Hammermeister KE, Grover F, Daley J. Independent association between acute renal failure and mortality following cardiac surgery. Am J Med 1998; 104:343-8. 